\title{
The Role of Radiological Parameters in Assessing Response to Neoadjuvant Therapy in Borderline Resectable Pancreatic Cancer
}

\author{
Nervana Hussien ${ }^{1}$, Kareem Sallam ${ }^{2}$, Mostafa Abdel-Kawi ${ }^{3}$, Mai Ezz El Din ${ }^{4}$ \\ ${ }^{1}$ Department of Clinical Oncology, Faculty of Medicine, Helwan University, Cairo, Egypt; ${ }^{2}$ Department \\ of Surgery, Faculty of Medicine, Helwan University, Cairo, Egypt; ${ }^{3}$ Department of Radiology, Faculty of \\ Medicine, Helwan University, Cairo, Egypt; ${ }^{4}$ Department of Clinical Oncology, Faculty of Medicine, Ain \\ Shams University, Cairo, Egypt.
}

Background: Surgery in pancreatic cancer remains the curative option, consequently drawing attention to the importance of an indeterminate group of patients potentially curable by undergoing this procedure, Borderline Resectable Pancreatic Cancer (BRPC). Despite this seemingly positive outlook the outcome of these patients remains undefined.

Aim: To assess the response of BRPC patients to neoadjuvant treatment and the accuracy of radiological constraints in their selection.

Methods: Data extraction from a university hospital filing system from September 2015 to September 2018 was performed to select patients with BRPC. The National Comprehensive Cancer Network (NCCN) criteria to define BRPC were used. Clinical, surgical and radiological parameters were collected pre/ post-operative for all cases and correlated to outcome.

Results: Sixty patients with BRPC were identified. The outcome of neoadjuvant treatment was partial response in $6(10 \%)$ patients, stable disease in $49(81.7 \%)$ and progressive disease in $5(8.3 \%)$. The majority $(78.3 \%)$ of patients underwent pancreatectomy. In patients who underwent resection a median overall survival of 31 months (95\% CI: 29.180 - 32.820) was achieved vs. 17 months (95\% CI: 15.625 - 18.375) in non-resected cases ( $<0.001)$. Response to neoadjuvant therapy was found to be favorable for overall survival $(p=0.014)$ and progression-free survival $(p=0.006)$. Response Evaluation Criteria in Solid Tumors (RECIST) criteria did not predict operative potential.

Conclusion: RECIST criteria lacked predictive potentiality for surgical intervention after neoadjuvant chemotherapy in a cohort of BRPC patients. The significant positive outcome observed in BRPC patients who underwent surgery emphasizes that the decision for this procedure should not depend on the response to neoadjuvant treatment per se.

Keywords: Borderline resectable pancreatic cancer, Neoadjuvant chemotherapy, Radiological assessment, RECIST criteria Corresponding author: Nervana Hussien, MD; Department of Clinical Oncology, Faculty of Medicine, Helwan University, Cairo, Egypt; Email: hassannervana5455@yahoo.com

Submitted: 8-November-2019, Revised: 9-January-2020, Accepted: 22-January-2020, Published online: 1-February-2020

(cc) BY

\section{INTRODUCTION}

Knowing the dismal prognosis of pancreatic cancer and the emergence of surgery with its limitations as the only possible curative intervention underscores the pressure experienced by the medical team to incorporate this modality whenever feasible. "Borderline resectable pancreatic cancer" (BRPC) is a grey zone that includes surgical candidates with a high risk of margin positivity (R1 or R2) ${ }^{1}$.

The National Comprehensive Cancer Network (NCCN) adopted the term "borderline resectable" in 2006 and various definitions followed, all based mainly on computerized tomography (CT) characterization of the disease and its surrounding vasculature ${ }^{2}$.

With the evolution in radiology and the expansion in vessel contact terminology together with advancement in surgical capabilities, a group of patients designated previously as unresectable were rendered potentially resectable. The advancements in systemic therapy and radiotherapy and their incorporation into the neoadjuvant scene further emphasize the need for a dynamic definition of BRPC ${ }^{3}$.

Taking these factors into consideration, patients meeting current BRPC definition criteria were reviewed retrospectively to analyze their treatment outcome and to examine the accuracy of radiological constraints in selecting them.

\section{METHODS}

A retrospective review of the medical records of patients with BRPC who were managed at Helwan and Ain Shams University hospitals from September 2015 to September 2018. The study was granted local Institutional Review Board approval. 
Borderline resectable pancreatic cancer was defined according to the NCCN criteria as any tumor radiologically in contact with major peripancreatic vasculature as the portal vein $(\mathrm{PV})$ or superior mesenteric vein $(\mathrm{SMV})$ that was deemed resectable (+/- reconstruction) or $<180^{\circ}$ involvement of the common hepatic artery (CHA) or superior mesenteric artery (SMA) without any tumour extension reaching the celiac axis (CA) or hepatic artery bifurcation. Metastatic, resectable, and locally advanced cases were excluded.

Data on treatment, response to neoadjuvant chemotherapy, pancreatic protocol CTs pre- and posttherapy, degree of resection, outcome and survival were collected.

All patients underwent dedicated pancreatic neoplasia staging before and after treatment that was performed by multi-slice CT using a row scanner (Philips ${ }^{\circledR}$ Ingenuity TF 128 multi detector) and a standard pancreatic protocol optimized for imaging such tumours. Water was given as a negative contrast to distend the stomach and duodenum. Iodinated contrast (100 to $150 \mathrm{ml})$ was injected intravenously directly prior to imaging at the rate of 5 $\mathrm{mL} / \mathrm{second}$. An early arterial phase of the study (delay 20 seconds post injection), a pancreatic parenchymal (early portal) phase achieved about 30 to 40 seconds after the onset of the intravenous contrast injection were acquired. Images were reconstructed in coronal, sagittal and oblique axial planes at 2-mm slice thickness to clarify the lesions from the surrounding vascular anatomy.

Multidisciplinary consultation was sought regarding subsequent management and resectability according to the patient's response and performance status. Response Evaluation Criteria in Solid Tumors (RECIST) version 1.1 was utilized to measure neoadjuvant therapy effect and pathological completeness of margins $(\mathrm{R})$ was reported in patients who underwent the procedure ${ }^{4}$.

Operative technique commenced with an abdominal exploration, pancreatic head mobilization and exploration of celiac/ hepatic artery and superior mesenteric artery proximal or distal to the site of presumed contact. This was followed by tracking down to the area of the contact to decide resectability. Portal vein involvement was managed by subadventitial dissection, partial circumference resection with vein patch, segmental resection with either end-to-end anastomosis or replacement by internal jugular vein graft. Portal vein involvement did not preclude surgery intraoperatively except in 2 situations:1- Portal vein/SMV thrombosis, 2-Involvement of proximal jejunal branches. Resectable cases underwent a pancreatectoduodenectomy with triple anastomosis. The configuration of anastomoses varied according to the preference of the operating team.

The primary objective was to measure resectability of BRPC and the accuracy of radiological evaluation in determining this. Secondary objectives were overall survival (OS) and Progression free survival (PFS) their relation to baseline characteristics. Overall survival was defined as the time interval between the date of diagnosis and the date of death. The definition of PFS was from the date of diagnosis till the date of cancer recurrence (surgical cases)/progression (non- surgical cases).

Survival rates were estimated and graphed using the Kaplan-Meier method. Log rank test was used to compare time-to-event variables by levels of a factor variable. Pearson's correlation was used to assess the correlation between numerical variables. A significance level of $\mathrm{P}<0.05$ was used in all tests. All statistical procedures were carried out using IBM Statistical Package for the Social Sciences (SPSS) version 20 for Windows.

\section{RESULTS}

A total of 60 cases who met the inclusion criteria were identified in the allotted time frame, with baseline characteristics displayed in table 1 .

Table 1. Patient's Baseline characteristics

\begin{tabular}{ll}
\hline Variable & Mean \pm SD \\
\hline Age in years & $55 \pm 10.6$ \\
\hline & No. $(\%)$ \\
\hline Sex & \\
\hline Male & $40(66.7)$ \\
\hline Female & $20(33.3)$ \\
\hline ECOG performance status & \\
\hline 0 & $30(50)$ \\
\hline 1 & $26(43.3)$ \\
\hline 3 & $3(5)$ \\
\hline Weight loss & $1(1.7)$ \\
\hline Abdominal pain & $35(58.3)$ \\
\hline Jaundice & $40(66.7)$ \\
\hline
\end{tabular}

\begin{tabular}{|c|c|}
\hline Tumor location & \\
\hline Head & $25(41.7)$ \\
\hline Uncinate & $5(8.3)$ \\
\hline Neck & $20(33.3)$ \\
\hline Body/tail & $10(16.7)$ \\
\hline \multicolumn{2}{|l|}{ Involvement of vessels by CT scan } \\
\hline Portal vein / superior mesenteric vein & $45(75)$ \\
\hline Superior mesenteric artery & $15(25)$ \\
\hline Common hepatic artery & $27(28.3)$ \\
\hline \multicolumn{2}{|l|}{ Neoadjuvant chemotherapy regimen } \\
\hline Gemcitabine-based & $35(58.3)$ \\
\hline \multirow[t]{2}{*}{ FOLFIRINOX protocol } & $25(41.7)$ \\
\hline & Median (range) \\
\hline CA19-9 at diagnosis $(\mathrm{U} / \mathrm{mL})$ & $120(40-330)$ \\
\hline Number of chemotherapy cycles & $4(2-6)$ \\
\hline
\end{tabular}

Partial response to neoadjuvant therapy was achieved in $6(10 \%)$ patients, progressive disease in $5(8.3 \%)$ and the majority $(49[81.7 \%])$ attained stable disease status.

Surgical resection was performed in $47(78.3 \%)$ BRPC patients. The remaining $13(21.7 \%)$ non- surgical cases were not operated due to poor performance in 5 patients, disease progression in 5 cases and refusal in 3 patients. Of 
the 47 surgical candidates, an R0 was achieved in 40 $(85.1 \%)$ and $\mathrm{R} 1$ in the remaining 7 (14.9\%).

Of the 40 R0 surgery cases 6 had achieved a partial response to neoadjuvant chemotherapy and the remaining 34 stable disease, whilst all 7 R1 patients had stable disease on preoperative $\mathrm{CT}$ assessment. A total of 38 cases had died by the end of the study period $(63.3 \%)$ whilst $22(36.7 \%)$ were alive. Nineteen $(40.4 \%)$ patients remained free of disease recurrence but $28(59.6 \%)$ cases eventually recurred.

A median OS of 30 months (95\% Confidence Interval [CI]: 28.047-31.953) for the whole cohort was observed. The median OS was 32 months (95\% CI: 24.774 - 39.226) for resected BRPC patients compared to 17 months $(95 \%$ CI: 15.854 - 18.146) in non-surgical candidates $(p<0.001)$ as evident in figure 1 . The median PFS for the cohort as a whole was 21 months (95\% CI: 20.36 - 22.46) and it was significantly $(p<0.001)$ longer in patients who underwent surgery further than those who did not (22 months [95\% CI: 20.732 - 23.268] vs. 3 months [95\% CI: 2.35 - 3.58], respectively)

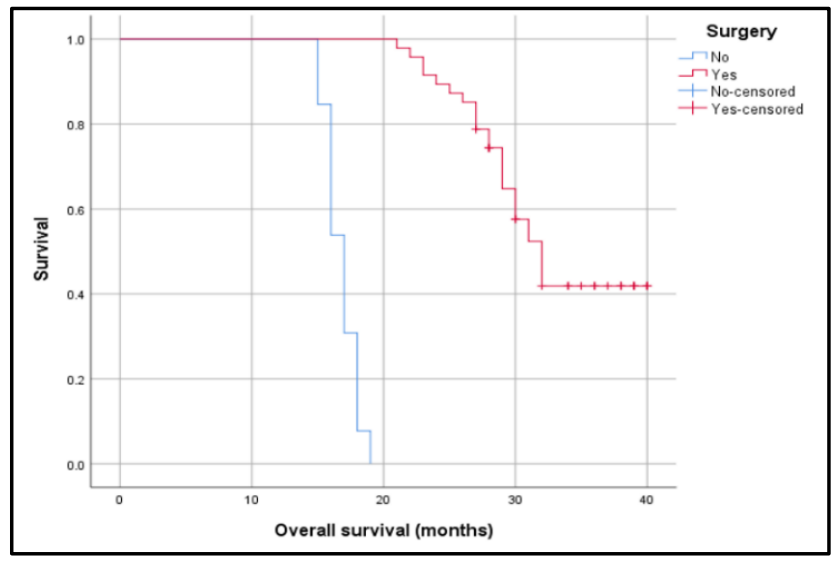

Figure 1. Overall survival curves according to surgical resection

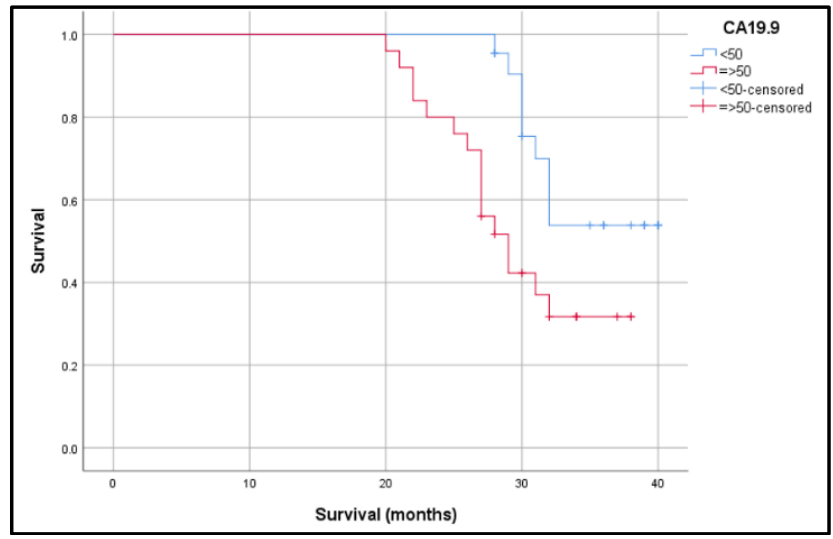

Figure 2. Overall survival curves according to CA 19.9 levels

A positive correlation between response to neoadjuvant therapy (PR) and outcome was observed for median OS (not reached in responders vs. 32 months [95\%CI: $30.333-$ 33.667] in non-responders, $\mathrm{p}=0.014$ ) and median PFS (not reached in responders vs. 22 months [95\% CI: 20.732 23.268] in non-responders, $\mathrm{p}=0.006)$. Resected BRPC with SD by CT criteria achieved an OS of 31 months (95\% CI: 29.180 - 32.820) vs. unresected with SD 17 months (95\% CI: 15.625-18.375) ( $\mathrm{p}<0.001)$. Low and high levels of CA19.9, set at $50 \mathrm{U} / \mathrm{mL}$ or less as low, was also related to outcome as the median OS for low CA19.9 was not reached vs. 29 months (95\% CI: 26.086 - 31.914) in high CA19.9 (p $=0.008$ ) (figure 2).

\section{DISCUSSION}

With 458,918 new cases / year reported by GLOBOCAN 2018, pancreatic cancer ranks as number 14 in the list of all cancers combined. The small difference between the number of newly diagnosed cases and deaths (432.242 deaths / year) caused by pancreatic cancer indicates a dismal prognosis faced by those diagnosed with this illness ${ }^{5}$.

Surgical resection remains the only potentially curative therapy for pancreatic cancer with a 5-year survival rate of $15-25 \%$; hence, arose the need to better define the BRPC category in order to take advantage of this modality in improving outcome, even if at a modest rate ${ }^{6,7}$.

The surgical resection quality is an important prognostic guide to expectant survival as achieving complete resection (R0) is significantly better than with an R1 (residual tumor cells on resection margins) or $\mathrm{R} 2$ (macroscopic residual tumor cells) resection. In fact, incomplete (R1 or R2) resections concur a somewhat lower survival rate than after radio-chemotherapy without surgery ${ }^{8}$. The concept of neoadjuvant therapy in BRPC aspires to render patients suitable for the procedure with the minimal or preferably nil R status 9 .

In the real world, to be surgically designated as BRPC is based entirely on anatomic criteria, and many have questioned the lack of standardization in categorizing this subgroup ${ }^{10}$. In the era of genomic profiling neglecting to incorporate the biological behavior of the cancer coupled with a sound assessment to determine the appropriateness of resection regarding the patient's ability to endure the physiological strain of surgery seems irrational ${ }^{10}$. The first mention of biological and conditional criteria for resectability was by Katz et al in 2008 but these have not been integrated into definitions of BRPC. The basic biologic criteria back then were the presence of metastasis and conditional criteria was the existence of significant medical comorbidities and/or poor performance status ${ }^{11}$.

In the current study resection was performed for $78 \%$ of BRPC patients, and in $40(85.1 \%)$ an $\mathrm{R} 0$ resection was achieved. This is slightly higher than reported in the ALLIANCE trial A021101 (68\%) ${ }^{12}$, PREOPANC-1 trial $(72 \%)^{13}$ and a meta-analysis on 935 BRPC patients $(69 \%)$ 14.

Statistically significant improvement in terms of OS was attained for resected BRPC (32 months) in contrast to 
unresected patients (17 months). This is in agreement with the recent meta-analysis on BRPC subjects that registered OS of 27.4 months vs. 12.9 months in resectable and unresectable cases respectively ${ }^{15}$. Other series reported lower survival rates ${ }^{12,16}$.

This study mainly aimed to correlate current radiologic assessment parameters to chances of performing a margin free resection; R0 optimally or even an R1. Applying RECIST criteria, only $10 \%$ of patients achieved PR to neoadjuvant chemotherapy and the majority (82\%) achieved SD. However, $78 \%$ of patients underwent surgery and an R0 was achieved in $85 \%$ of them. This highlights the shortages of the RECIST criteria in determining resectability and predicting thereafter margin status, again signaling the dire need of more factors to help in categorizing these patients.

Surgical resection was performed for the majority $(78 \%)$ of BRPC patients in the current study and in a minority $(22 \%)$ it was not performed. Of those who underwent surgery, an R0 was achieved in $85 \%$ and R1 in the remaining $15 \%$. This is similar to the findings of other studies that have evaluated the sensitivity of imaging to determine resectability in these patients ${ }^{17,18}$.

The use of an imperfect marker such as CA19-9, which may be used as a surrogate of the biological disease burden may aid in classifying this distinct category of patients further. It was observed that patients having CA19-9 levels exceeding $50 \mathrm{U} / \mathrm{mL}$ had inferior survival rate compared to those with low CA19-9. A consensus statement issued by the International Association of Pancreatology (IAP) in 2016 concerning a Biological definition of BRPC suggested including a CA 19.9 level more than 500 units $/ \mathrm{ml}^{10}$. Additionally, the American Society of Clinical Oncology (ASCO) clinical practice guidelines found a relationship between CA19-9 levels and tumor resectability and prognosis in $>1000$ patients who underwent surgery for potentially curable pancreatic cancer. They found patients with low CA 19-9 levels from 5 - <37 to have a resectability rate of $79.7 \%$ and a 28.5 months median survival time (MST) after resection. Those with levels $37-<100$ had $83.3 \%$ resectability rate and 26.9 months MST 26.9, and those with levels $100-<250$ had $82.2 \%$ resectability rate and 22.5 months MST, and so on till the highest CA19-9 $1000-<2000$ level with the lowest resectability rate of $61.1 \%$ and MST of 1 year ${ }^{19}$.

Despite our small sample size these consistent observations correlate well with the generally low CA19-9 level (120 U/mL, range 40-330) observed in the current study and thus fortifying the biological concept of BRPC and serving as a logical interpretation to our resection rate and median OS.

Attainment of a response in fact performs as a biological marker worthy of incorporation into the current algorithm of BRPC subset as evident from the better outcome seen in the limited cases with PR vs. those with SD.

The primary limitation of this study is the selection bias inherent in retrospective observations. Second, full pathological parameters such as lymph node involvement for example were not accounted for. Finally, the small sample size made it difficult to come to firm conclusions.

Yet, it is despite this limited sample that previous concepts in the categorization of this unique entity have been replayed and the whole series served as a portrayal of the biological concept by its low overall CA19-9 levels and favorable outcomes. It challenged the anatomical constraints of definition along with their equivalent measuring counterparts and urged a broader incorporation of factors into the defining equation of potentially resectable pancreatic cancer ${ }^{10,19}$, urging the search for more defining measures of response as for example the Choi criteria in gastrointestinal stromal tumors ${ }^{20}$.

So, even this small number of BRPC patients represents an acceptable single series depiction of a rare subentity of this usually late stage presenting disease.

To conclude, even with a predominantly SD outcome in $81.7 \%$ of cases, the mere ability to be able to undergo surgery resulted in an $\mathrm{R} 0$ resection for $56.7 \%$ coupled with an improved OS. Seemingly, standard response assessment criteria for patients with BRPC after neoadjuvant chemotherapy should not deprive them of a surgical intervention if they lack a favorable result, yet remain fit enough for the procedure. Omitting the surgical "opportunity" could unjustly deprive them a chance for improved survival in this inherently miserable disease.

\section{CONFLICTS OF INTEREST}

The authors have no conflict of interest to declare.

\section{REFERENCES}

1. Mehta VK, Fisher G, Ford JA, et al. Preoperative chemoradiation for marginally resectable adenocarcinoma of the pancreas. J Gastrointest Surg. 2001; 5(1): 27-35.

2. Hough TJ, Raptopoulos V, Siewert B, Matthews JB. Teardrop superior mesenteric vein: CT sign for unresectable carcinoma of the pancreas. AJR Am J Roentgenol. 1999; 173(6): 15091512.

3. Cassinotto C, Sa-Cunha A, Trillaud H. Radiological evaluation of response to neoadjuvant treatment in pancreatic cancer. Diagn Interv Imaging. 2016; 97(12): 1225-1232.

4. Eisenhauer EA, Therasse P, Bogaerts J, et al. New response evaluation criteria in solid tumours: revised RECIST guideline (version 1.1). Eur J Cancer. 2009; 45(2): 228-247.

5. Bray F, Ferlay J, Soerjomataram I, Siegel RL, Torre LA, Jemal A. Global Cancer Statistics 2018: GLOBOCAN Estimates of Incidence and Mortality Worldwide for 36 Cancers in 185 Countries. CA Cancer J Clin. 2018; 68(6): 394-424.

6. Sohn TA, Yeo CJ, Cameron JL, et al. Resected adenocarcinoma of the pancreas - 616 patients: results, outcomes, and prognostic indicators. J Gastrointest Surg. 2000; 4(6): 567-579.

7. Neoptolemos JP, Stocken DD, Dunn JA, et al. Influence of resection margins on survival for patients with pancreatic cancer treated by adjuvant chemoradiation and/or chemotherapy in the ESPAC-1 randomized controlled trial. Ann Surg. 2001; 234(6): 758-768.

8. Bilimoria KY, Talamonti MS, Sener SF, et al. Effect of hospital volume on margin status after 
pancreaticoduodenectomy for cancer. J Am Coll Surg. 2008; 207(4): 510-519.

9. Katz MH, Marsh R, Herman JM, et al. Borderline resectable pancreatic cancer: Need for standardization and methods for optimal clinical trial design. Ann Surg Oncol. 2013; 20(8): 2787-2795.

10. Isaji S, Mizuno S, Windsor JA, et al. International consensus on definition and criteria of borderline resectable pancreatic ductal adenocarcinoma 2017. Pancreatology. 2018; 18(1): 2 11.

11. Katz MH, Pisters PW, Evans DB, et al. Borderline resectable pancreatic cancer: the importance of this emerging stage of disease. J Am Coll Surg. 2008; 206(5): 833-846.

12. Katz MH, Shi Q, Ahmad SA, et al. Preoperative Modified FOLFIRINOX Treatment Followed by Capecitabine-Based Chemoradiation for Borderline Resectable Pancreatic Cancer: Alliance for Clinical Trials in Oncology Trial A021101. JAMA Surg. 2016; 151(8): e161137.

13. Quan K, Sutera P, Xu K, et al. Results of a prospective phase 2 clinical trial of induction gemcitabine/capecitabine followed by stereotactic ablative radiation therapy in borderline resectable or locally advanced pancreatic adenocarcinoma. Pract Radiat Oncol. 2018; 8(2): 95-106.

14. Dhir M, Malhotra GK, Sohal DPS, et al. Neoadjuvant treatment of pancreatic adenocarcinoma: a systematic review and meta-analysis of 5520 patients. World J Surg Oncol. 2017; 15(1): 183 .

15. Javed AA, Wright MJ, Siddique A, et al. Outcome of Patients with Borderline Resectable Pancreatic Cancer in the Contemporary Era of Neoadjuvant Chemotherapy. J Gastrointest Surg. 2019; 23(1): 112-121.

16. Kim HS, Jang JY, Han Y, et al. Survival outcome and prognostic factors of neoadjuvant treatment followed by resection for borderline resectable pancreatic cancer. Ann Surg Treat Res. 2017; 93(4): 186-194.

17. Shrestha B, Sun Y, Faisal F, et al. Long-term survival benefit of upfront chemotherapy in patients with newly diagnosed borderline resectable pancreatic cancer. Cancer Med. 2017; 6(7): 1552-1562.

18. Joo I, Lee JM, Lee ES, et al. Preoperative MDCT assessment of resectability in borderline resectable pancreatic cancer: Effect of neoadjuvant chemoradiation therapy. AJR Am J Roentgenol. 2018; 210(5): 1059-1065.

19. Khorana AA, Mangu PB, Berlin J, et al. Potentially curable pancreatic cancer: American Society of Clinical Oncology clinical practice guideline. J Clin Oncol. 2016; 34(21): 25412556.

20. Choi H. Response evaluation of gastrointestinal stromal tumors. Oncologist. 2008; 13(Suppl 2): 4-7. 